\title{
Effects of nitrogen incorporation on a direction of a surface band bending investigated by polarity of terahertz electromagnetic waves in GaAs 1-x $\mathrm{N}_{\mathrm{x}}$ epitaxial layers
}

Hideo Takeuchi, Junichi Yanagisawa, Jun Hashimoto, Masaaki Nakayama

\begin{tabular}{|c|l|}
\hline Citation & Journal of Applied Physics, 105(9); 093539 \\
\hline Issue Date & 2009-05-14 \\
\hline Type & Journal Article \\
\hline Textversion & Publisher \\
\hline Right & $\begin{array}{l}\text { C 2009 American Institute of Physics. This article may be downloaded for personal } \\
\text { use only. Any other use requires prior permission of the author and AIP Publishing. } \\
\text { The following article appeared in Journal of Applied Physics and maybe found at } \\
\text { https://doi.org/10.1063/1.3125451 }\end{array}$ \\
\hline DOI & \begin{tabular}{l}
$10.1063 / 1.3125451$ \\
\hline
\end{tabular} \\
\hline
\end{tabular}

Self-Archiving by Author(s)

Placed on: Osaka City University Repository 


\title{
Effects of nitrogen incorporation on a direction of a surface band bending investigated by polarity of terahertz electromagnetic waves in $\mathrm{GaAs}_{1-x} \mathrm{~N}_{\boldsymbol{x}}$ epitaxial layers
}

\author{
Hideo Takeuchi, ${ }^{1, a)}$ Junichi Yanagisawa, ${ }^{1}$ Jun Hashimoto, ${ }^{2}$ and Masaaki Nakayama ${ }^{2}$ \\ ${ }^{1}$ Department of Electronic Systems Engineering, School of Engineering, The University of Shiga Prefecture, \\ 2500 Hassaka-cho, Hikone, Shiga 522-8533, Japan \\ ${ }^{2}$ Department of Applied Physics, Graduate School of Engineering, Osaka City University, 3-3-138 \\ Sugimoto, Sumiyoshi-ku, Osaka 558-8585, Japan
}

(Received 27 January 2009; accepted 30 March 2009; published online 14 May 2009)

\begin{abstract}
We have investigated the polarity of the terahertz electromagnetic wave from $\mathrm{GaAs}_{1-x} \mathrm{~N}_{x}$ epitaxial layers with $x=0.43 \%$ and $1.53 \%$ in order to clarify the effects of nitrogen incorporation on the direction of the surface band bending using an undoped GaAs/n-type GaAs( $i$-GaAs $/ n$-GaAs) epitaxial layer structure and a semi-insulating GaAs (SI-GaAs) crystal as reference samples. A numerical calculation on the basis of the Boltzmann-Poisson model clarifies that the conduction band of the $i$-GaAs $/ n$-GaAs sample bends upward at the surface region, which indicates that photogenerated electrons flow into the inside. In the measurement of the terahertz wave, it is observed that the polarity of the terahertz wave from the SI-GaAs crystal is the same as that of the $i$-GaAs/n-GaAs sample, namely, the present SI-GaAs crystal has an upward band bending. In contrast, the terahertz-wave polarity is reversed in the $\mathrm{GaAs}_{1-x} \mathrm{~N}_{x}$ samples in spite of the relatively small nitrogen mole fraction; namely, the $\mathrm{GaAs}_{1-x} \mathrm{~N}_{x}$ samples have a downward band bending. The above-mentioned terahertz-wave polarity reversal is attributed to the phenomenon that conduction-band bottom is considerably lowered by the band anticrossing peculiar to $\mathrm{GaAs}_{1-x} \mathrm{~N}_{x}$, which results in approaching the conduction-band bottom to the surface Fermi level. This modifies the direction of the surface band bending connected with the polarity of the terahertz wave.
\end{abstract}

(C) 2009 American Institute of Physics. [DOI: 10.1063/1.3125451]

\section{INTRODUCTION}

Compound semiconductors, by being irradiated by femtosecond-laser pulses, emit a terahertz electromagnetic wave originating from a surge current in the surface band bending region. It has been considered that the abovementioned phenomenon can be applied to a compact and convenient terahertz emitter free from a device fabrication. ${ }^{1}$ Accordingly, the terahertz emission from a compound semiconductor surface has been investigated from the viewpoint of achieving more intense terahertz emission. As a result of exploring suitable materials, it is found that InAs single crystals are good candidates for the terahertz emitters., ${ }^{2,3}$ Recently, we proposed that the design of an epitaxial layer structure, e.g., undoped GaAs/n-type GaAs( $i$-GaAs $/ n$-GaAs) structure, is an important strategy for enhancing the terahertz emission intensity. ${ }^{4}$

As mentioned above, in the research field of the terahertz emission from compound semiconductors, most of the efforts have been focused on finding more intense terahertz emitters. However, the analysis of the characteristics of the emitted terahertz waveform, e.g., the polarity, has been little considered. The polarity of the emitted terahertz wave reflects the direction of the surface band bending that determines the direction of the surge current of photogenerated carriers around the surface. In the case where the conduction band bends upward at the surface region, the electrons flow into

\footnotetext{
${ }^{a)}$ Electronic mail: takeuchi.h@e.usp.ac.jp.
}

the internal side of a crystal. In the case where the conduction band bends downward at the surface region, the electrons flow into the surface side of a crystal. The change in an electron (or hole) flow direction causes the reversal of the polarity of the terahertz wave. Thus, the investigation of the polarity of the terahertz wave is applicable to clarify the direction of the surface band bending.

In the present work, we investigated the surface band bending of $\mathrm{GaAs}_{1-x} \mathrm{~N}_{x}$ epitaxial layers by analyzing the polarity of the terahertz wave from the samples. $\mathrm{GaAs}_{1-x} \mathrm{~N}_{x}$ is a III-V dilute nitride that exhibits giant bowing of the bandgap energy as a function of nitrogen content: a reduction in the band-gap energy by $180 \mathrm{meV}$ per nitrogen mole fraction of $1 \% .^{5}$ It is, therefore, scientifically interesting to investigate whether the surface band bending, which is dominated by numerous midgap levels on the surface, is influenced by the incorporation of small mole fraction of nitrogen to GaAs. The information on the surface band bending is also technologically useful to form a metal electrode on the $\mathrm{GaAs}_{1-x} \mathrm{~N}_{x}$ epitaxial layer.

The present paper is organized as follows. Initially, we introduce an $i$-GaAs $/ n$-GaAs epitaxial layer structure and demonstrate the appropriateness as a reference sample from the viewpoint of the surface potential structure. Next, we demonstrate the terahertz waveforms from the $i$-GaAs /n-GaAs structure, semi-insulating GaAs (SI-GaAs) crystal, and undoped $\mathrm{GaAs}_{1-x} \mathrm{~N}_{x}$ epitaxial layers with $x=0.43 \%$ and $1.53 \%$. It is found that the nitrogen incorporation causes the 


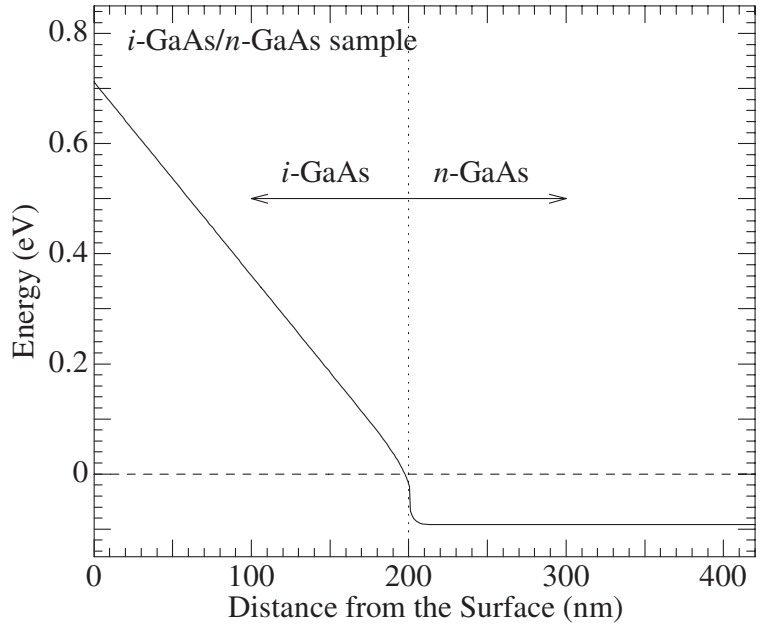

FIG. 1. Potential energy of the $i$-GaAs $(200 \mathrm{~nm}) / n$-GaAs $(3 \mu \mathrm{m}, 3$ $\times 10^{18} \mathrm{~cm}^{-3}$ ) epitaxial layer structure as a function of distance from the surface calculated on the basis of the Boltzmann-Poisson model. The solid and dashed lines indicate the conduction-band energy and Fermi level, respectively.

reversal of the terahertz-wave polarity, which suggests the change in a surface band bending in the $\mathrm{GaAs}_{1-x} \mathrm{~N}_{x}$ samples. Finally, we discuss the mechanism of the change in the surface band bending on the basis of a band anticrossing model for III-V dilute nitrides. ${ }^{6,7}$

\section{POTENTIAL STRUCTURE OF THE $i$-GaAs/n-GaAs EPITAXIAL LAYER STRUCTURE}

In order to clarify the properties of the $i$-GaAs $/ n$-GaAs epitaxial layer structure that is a reference sample, we numerically calculated the equilibrium potential structure in the $i$-GaAs $(200 \mathrm{~nm}) / n$-GaAs $\left(3 \mu \mathrm{m}, 3 \times 10^{18} \mathrm{~cm}^{-3}\right)$ sample on the basis of the Boltzmann-Poisson model, ${ }^{8,9}$ where the values in the parentheses denote the individual layer thickness and doping concentration. The parameters used in the calculations are the same as those used in Ref. 10. It should be noted in advance to the discussion in Sec. IV that the surface Fermi level of $i$-GaAs locates at almost the center of the band gap. ${ }^{10,11}$ The results of the above-mentioned calculation are shown in Fig. 1. In Fig. 1, the solid line denotes the conduction-band energy as a function of distance from the surface, where the origin of the energy axis corresponds to the Fermi level (dashed line). The conduction-band energy has a finite potential slope in the $i$-GaAs layer induced by the surface Fermi level pinning, which produces a uniform built-in electric field of $35 \mathrm{kV} / \mathrm{cm}$ in the $i$-GaAs layer. Such a high electric field is sufficient to cause a surge current induced by the drift motion because the electron velocity saturates in the range above about $10 \mathrm{kV} / \mathrm{cm} .{ }^{12}$ Consequently, the electrons generated in the $i$-GaAs layer flow into the $n$-GaAs underlayer, while the holes generated in the $i$-GaAs layer flow into the $i$-GaAs surface side. As a result, it is evident that the surface surge current, which is a sum of the electron and hole fluxes, flows into the $i$-GaAs surface side. The above-mentioned current flow enables the

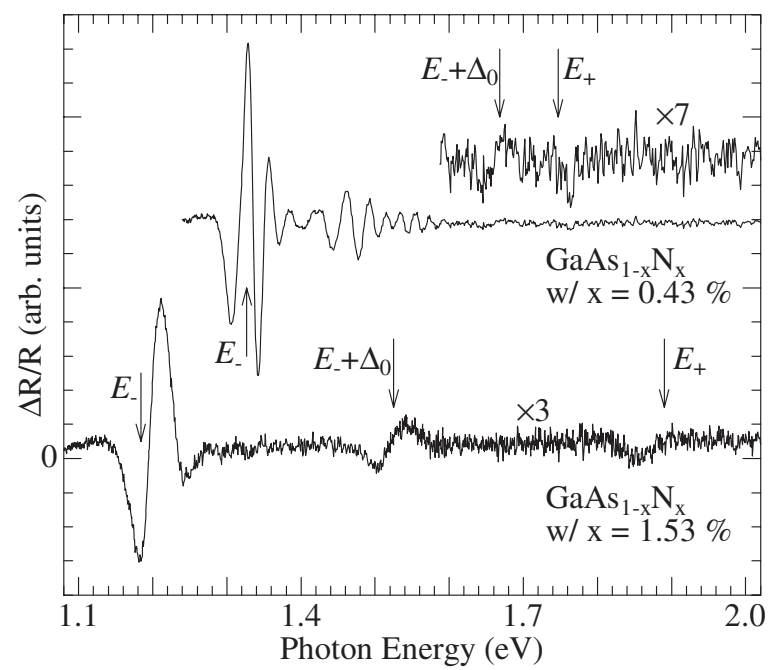

FIG. 2. PR spectra of the $\mathrm{GaAs}_{1-x} \mathrm{~N}_{x}(x=0.43 \%$ and $1.53 \%)$ samples at room temperature. The position of the arrow labeled $E_{-}\left(E_{+}\right)$corresponds to the energy of the transition between the lower (higher) conduction subband and heavy hole and/or light hole bands at the $\Gamma$-point, and the position of the arrow labeled $E_{-}+\Delta_{0}$ corresponds to the $E_{-}$-subband-split-off-hole-band transition at the $\Gamma$-point. For clarity, each spectrum is vertically shifted.

$i$-GaAs/n-GaAs epitaxial layer structure to be a reference sample to check the polarity of the terahertz electromagnetic waves.

\section{SAMPLES AND EXPERIMENTAL PROCEDURE}

The samples were a (001)-oriented SI-GaAs crystal doped with $\mathrm{CrO}$ and undoped $\mathrm{GaAs}_{1-x} \mathrm{~N}_{x}$ epitaxial layers with $x=0.43 \%$ and $1.53 \%$. The $i-\operatorname{GaAs}(200 \mathrm{~nm}) / n$-GaAs ( $3 \mu \mathrm{m}, 3 \times 10^{18} \mathrm{~cm}^{-3}$ ) epitaxial layer structure was also used as a reference sample. The thickness of the $\mathrm{GaAs}_{1-x} \mathrm{~N}_{x}$ layer of each sample was $500 \mathrm{~nm}$. The samples of the epitaxial layer structures were grown by metal organic vapor phase epitaxy. The $i$-GaAs $/ n$-GaAs sample was grown on a $2^{\circ}$-off (001) SI-GaAs substrate, while the $\mathrm{GaAs}_{1-x} \mathrm{~N}_{x}$ samples were grown on a (001) SI-GaAs substrate. We confirmed the nitrogen incorporation in the $\mathrm{GaAs}_{1-x} \mathrm{~N}_{x}$ samples by measuring photoreflectance (PR) spectra. Figure 2 shows the PR spectra of the $\mathrm{GaAs}_{1-x} \mathrm{~N}_{x}$ samples at room temperature, where the pump-beam energy and power were $2.41 \mathrm{eV}$ and $1 \mathrm{~mW} / \mathrm{cm}^{2}$. The position of the arrow labeled $E_{-}\left(E_{+}\right)$ corresponds to the energy of the transition between the lower (upper) conduction subband and heavy hole and/or light hole bands at the $\Gamma$-point, and the position of the arrow labeled $E_{-}+\Delta_{0}$ corresponds to the $E_{-}$-subband-split-off-hole-band transition at the $\Gamma$-point. The energies of $E_{-}$and $E_{+}$are calculated using the following equation based on the band anticrossing model: ${ }^{6,7}$

$$
E_{ \pm}=\frac{1}{2}\left[\left(E_{g, \mathrm{GaAs}}+E_{N}\right) \pm \sqrt{\left(E_{g, \mathrm{GaAs}}-E_{N}\right)^{2}+4 x C_{\mathrm{GaAs}, N}^{2}}\right] .
$$

Here, the quantities of $E_{g, \mathrm{GaAs}}, E_{N}$, and $C_{\mathrm{GaAs}, N}$ are the fundamental transition energy of GaAs, the energy position of nitrogen-related level, and the hybridization matrix element, respectively. The values of 1.424 (Ref. 13), 1.65 , and $2.7 \mathrm{eV}$ (Ref. 14) are used for $E_{g, \mathrm{GaAs}}, E_{N}$, and $C_{\mathrm{GaAs}, N}$, respectively. 


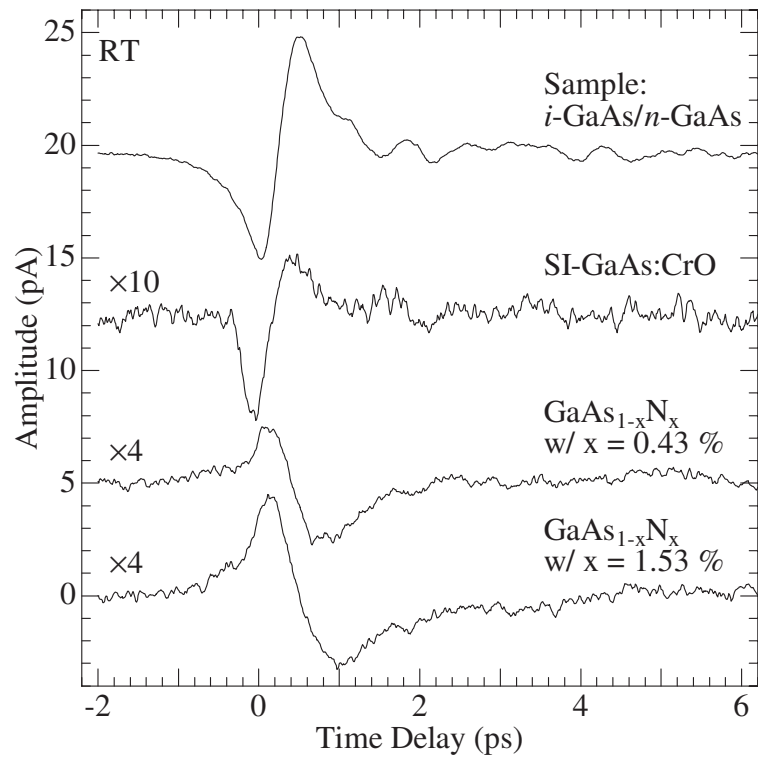

FIG. 3. Amplitudes of the terahertz electromagnetic waveforms of the $i$-GaAs $(200 \mathrm{~nm}) / n$-GaAs $\left(3 \mu \mathrm{m}, \quad 3 \times 10^{18} \mathrm{~cm}^{-3}\right), \quad$ SI-GaAs, and $\operatorname{GaAs}_{1-x} \mathrm{~N}_{x}(x=0.43 \%$ and $1.53 \%)$ samples as a function of time delay at room temperature. The pump-beam power was $40 \mathrm{~mW}$. The waveforms of the $\operatorname{GaAs}_{1-x} \mathrm{~N}_{x}(x=0.43 \%)$, SI-GaAs, and $i$-GaAs $/ n$-GaAs samples are vertically shifted by 5,12 , and $20 \mathrm{pA}$, respectively.

For the transition energy of $E_{-}+\Delta_{0}$, the value of $0.341 \mathrm{eV}$ (Ref. 13) was employed for the spin-orbit-coupling induced split-off energy of GaAs. At the transition energies calculated for $E_{+}, E_{-}$, and $E_{-}+\Delta_{0}$, the PR spectra exhibit the characteristic patterns. This fact indicates that the $\mathrm{GaAs}_{1-x} \mathrm{~N}_{x}$ epitaxial layers are formed with reasonable accuracy in nitrogen mole fraction.

The time-domain terahertz emission signals from the samples were measured with use of laser pulses with a duration time of about $70 \mathrm{fs}$. The repetition rate of the laser pulse was $90 \mathrm{MHz}$. The pump beam was focused on the sample with the angle of incidence of $45^{\circ}$. The diameter of the spot was $100 \mu \mathrm{m}$. The emitted terahertz beam was collected with use of two off-axis parabolic mirrors and was received by an optically gated bow tie antenna with a gap of $5.0 \mu \mathrm{m}$ formed in a low-temperature-grown GaAs. The terahertz beams illuminated the front side of the antenna so that a $\mathrm{Si}$ hemispherical lens, which is sometimes employed as one of terahertz optics in the back illumination configuration, was needless. The powers of the pump and gate beams were fixed to 40 and $4.0 \mathrm{~mW}$, respectively. The wavelengths of the pump and gate beams were the same: $800 \mathrm{~nm}$. All the measurements were performed in the air at room temperature. The humidity was confirmed to be constant during the measurement. All the terahertz waveforms were therefore obtained under the same condition.

\section{RESULTS AND DISCUSSION OF TERAHERTZ WAVES}

Figure 3 shows the waveforms of the terahertz emission of the $i$-GaAs/n-GaAs, SI-GaAs, and $\mathrm{GaAs}_{1-x} \mathrm{~N}_{x}$ samples at the pump-beam power of $40 \mathrm{~mW}$. All the samples show single oscillation around the time delay of $0 \mathrm{ps}$, the so-called first burst. It is obvious that the amplitude of the first burst of the $i$-GaAs/ $n$-GaAs sample is the largest of all the samples, which originates both from the electric field sufficient to launch the drift current and from the completely depleted relatively thick $i$-GaAs layer. The details of characteristics of the terahertz emission from the $i$-GaAs/ $n$-GaAs sample have already been described in Ref. 4. As the time delay increases, the polarity of terahertz wave from the $i$-GaAs/ $n$-GaAs sample changes from the negative to the positive. The terahertz-wave polarity of the SI-GaAs sample is the same as that of the $i$-GaAs/n-GaAs sample, which indicates that the direction of the photogenerated current producing the terahertz wave is the same between the $i$-GaAs/n-GaAs and SIGaAs samples. Accordingly, it is considered that the present SI-GaAs sample has an upward band bending at the surface region. As shown in Fig. 3, the first burst amplitude of the SI-GaAs sample is the smallest, which suggests that the surface band bending is relatively small.

In contrast, the terahertz-wave polarities of the $\mathrm{GaAs}_{1-x} \mathrm{~N}_{x}$ samples are reversed compared with that of the $i$-GaAs/n-GaAs sample, which means that the photogenerated current direction in the $\mathrm{GaAs}_{1-x} \mathrm{~N}_{x}$ samples is opposite to that of the $i$-GaAs $/ n$-GaAs sample. The $\mathrm{GaAs}_{1-x} \mathrm{~N}_{x}$ sample, therefore, has a downward band bending at the surface region. It is noteworthy that even in the $\mathrm{GaAs}_{1-x} \mathrm{~N}_{x}$ sample with $x=0.43 \%$, the polarity is inverted compared with that of the $i$-GaAs $/ n$-GaAs sample. This indicates that the incorporation of the small amount of nitrogen can change the direction of the surface band bending.

Finally, we discuss the mechanism causing the reversal of the direction of the surface band bending in the $\mathrm{GaAs}_{1-x} \mathrm{~N}_{x}$ samples. In general, the surface Fermi level pinning originates from large amount of deep levels at the surface locating within the forbidden band. The electronic wave functions of the deep levels are strongly localized in the atomic-order region. In contrast, the average distance between the nitrogen atoms is estimated to be several ten nanometers in $\mathrm{a} \mathrm{GaAs}_{1-x} \mathrm{~N}_{x}$ epitaxial layer with $x=1 \%$, taking account of the fact that the atomic monolayer thickness is $0.283 \mathrm{~nm}$ for the (001) direction in GaAs. ${ }^{13}$ In addition, the energy of the nitrogen level locates above the conductionband bottom. These facts suggest that the nitrogen incorporation cannot disturb the electronic wave functions of the deep levels; therefore, the energies of the deep levels relative to the vacuum level are not influenced by the nitrogen incorporation.

The nitrogen incorporation, however, strongly affects the conduction band according to the band anticrossing model. In the framework of the band anticrossing model, the conduction band of GaAs and the energy level of the incorporated nitrogen strongly interact with each other as shown in Eq. (1) because the energy level of the incorporated nitrogen locates above the conduction-band bottom by $226 \mathrm{meV}$, where the value of $226 \mathrm{meV}$ corresponds to the energy difference between $E_{N}$ and the band-gap energy of GaAs. ${ }^{14}$ The above interaction generates the $E_{+}$and $E_{-}$subbands through the band anticrossing. The bottom energy of the $E_{-}$subband relative to the vacuum level becomes larger than the conduction bottom energy of GaAs relative to the vacuum level; namely, the $E_{-}$-subband bottom approaches the deep levels 
responsible for the surface Fermi level pinning. In contrast, in the inside far away from the surface, the Fermi level in $\mathrm{GaAs}_{1-x} \mathrm{~N}_{x}$ locates at almost the center of the band gap according to thermal statistical mechanics.

Here, we denote the difference in energy between the conduction-band bottom and the surface Fermi level as $\Delta \phi_{\text {surf }}$. In the case where the nitrogen incorporation causes the downward shift in the $E_{-}$subband, the quantity of $\Delta \phi_{\text {surf }}$ becomes smaller. In the same manner, we denote the energy difference between the conduction-band bottom and the Fermi level in the inside of the crystal as $\Delta \phi_{\text {in }}$. From the above discussion on the Fermi level in the inside of the crystal, it is apparent that $\Delta \phi_{\text {in }}$ is constant and that the value of $\Delta \phi_{\text {in }}$ is almost equal to the half of the band-gap energy. Thermal statistical mechanics tells us that at equilibrium, the Fermi level is uniform from the surface to the inside of the crystal. It should be reminded that the surface Fermi level locates at the center of the band gap in GaAs, which means that the surface band bending is negligibly small in a realistic GaAs crystal, $\Delta \phi_{\text {surf }}-\Delta \phi_{\text {in }} \approx 0$. In $\mathrm{GaAs}_{1-x} \mathrm{~N}_{x}$, even the small nitrogen incorporation makes the quantity $\Delta \phi_{\text {surf }}$ $-\Delta \phi_{\text {in }}$ negative owing to the band anticrossing producing $E_{-}$ subband in the conduction band, which bends the conduction band downward. Thus, it is concluded that the downward surface band bending in the $\mathrm{GaAs}_{1-x} \mathrm{~N}_{x}$ epitaxial layers results from the shift in the $E_{-}$subband toward the surface Fermi level at the surface region. This is the origin of the polarity reversal of the terahertz waves.

\section{SUMMARY}

We have investigated the polarity of the terahertz electromagnetic wave from the $i$-GaAs/n-GaAs epitaxial layer structure, SI-GaAs crystal, and $\mathrm{GaAs}_{1-x} \mathrm{~N}_{x}$ epitaxial layers with $x=0.43 \%$ and $1.53 \%$ in order to clarify the direction of the surface band bending. It has been observed that the terahertz-wave polarity of the SI-GaAs sample is the same as that of the $i$-GaAs $/ n$-GaAs sample. This result indicates that the present SI-GaAs sample has an upward surface band bending. In contrast, it has been found that the terahertzwave polarities of the $\mathrm{GaAs}_{1-x} \mathrm{~N}_{x}$ epitaxial layers are reversed compared with that of the $i$-GaAs/n-GaAs sample. The present finding indicates that the $\mathrm{GaAs}_{1-x} \mathrm{~N}_{x}$ epitaxial layers have a downward surface band bending. The mechanism of the downward surface band bending has been discussed on the basis of the band anticrossing model. The downward surface band bending in the $\mathrm{GaAs}_{1-x} \mathrm{~N}_{x}$ epitaxial layers is attributed to the phenomenon that the $E_{-}$subband at the surface region shifts toward the surface Fermi level owing to the band anticrossing.

\section{ACKNOWLEDGMENTS}

The samples of $\mathrm{GaAs}_{1-x} \mathrm{~N}_{x}$ epitaxial layers were provided from Sumitomo Electric Industries. The authors acknowledge Junpei Oda in Osaka City University for his assistance in the terahertz spectroscopic measurement.

${ }^{1}$ P. Gu and M. Tani, Terahertz Optoelectronics, edited by K. Sakai (Springer-Verlag, Berlin, 2005), pp. 63-97.

${ }^{2}$ X.-C. Zhang and D. H. Auston, J. Appl. Phys. 71, 326 (1992).

${ }^{3}$ N. Sarukura, H. Ohtake, S. Izumida, and Z. Liu, J. Appl. Phys. 84, 654 (1998).

${ }^{4}$ H. Takeuchi, J. Yanagisawa, T. Hasegawa, and M. Nakayama, Appl. Phys. Lett. 93, 081916 (2008).

${ }^{5}$ Dilute III-V Nitride Semiconductors and Material Systems, edited by A. Erol (Springer, Berlin, 2008).

${ }^{6}$ W. Shan, W. Walukiewicz, J. W. Ager III, E. E. Haller, J. F. Geisz, D. J. Friedman, J. M. Olson, and S. R. Kurz, Phys. Rev. Lett. 82, 1221 (1999).

${ }^{7}$ W. Shan, W. Walukiewicz, K. M. Yu, J. W. Ager III, E. E. Haller, J. F. Geisz, D. J. Friedman, J. M. Olson, S. R. Kurz, and C. Nauka, Phys. Rev. B 62, 4211 (2000).

${ }^{8}$ P. A. Basore, IEEE Trans. Electron Devices 37, 337 (1990).

${ }^{9}$ D. A. Clugston and P. A. Basore, Conference Records of the 26th IEEE Photovoltaic Specialists Conference, (IEEE, Piscataway, NJ, 1997) p. 207.

${ }^{10}$ H. Takeuchi, Y. Kamo, Y. Yamamoto, T. Oku, M. Totsuka, and M. Nakayama, J. Appl. Phys. 97, 063708 (2005).

${ }^{11}$ H. Shen, M. Dutta, L. Fotiadis, P. G. Newman, R. P. Moerkirk, W. H. Chang, and R. N. Sacks, Appl. Phys. Lett. 57, 2118 (1990).

${ }^{12}$ J. S. Blakemore, J. Appl. Phys. 53, R123 (1982).

${ }^{13}$ Semiconductors-Basic Data, edited by O. Madelung (Springer, Berlin, 1996).

${ }^{14}$ Dilute III-V Nitride Semiconductors and Material Systems, edited by A. Erol (Springer, Berlin, 2008) p. 129. 